\title{
OPTIMIZATION OF PUMP OPERATIONS IN A COMPLEX WATER SUPPLY NETWORK: NEW GENETIC ALGORITHM FRAMEWORKS
}

\author{
D. DE WRACHIEN ${ }^{1}$, S. MAMBRETTI ${ }^{2} \&$ E. ORSI ${ }^{2}$ \\ ${ }^{1}$ State University of Milano, Italy \\ ${ }^{2}$ Politecnico di Milano, Italy
}

\begin{abstract}
In previous papers, a simple genetic algorithm (GA) was developed for the optimization of pump operations in water-distribution networks. Its application at the water supply network of Milano showed the possibility of a great improvement of its performance in terms of both energy and economic savings. In the present paper is now investigated the possibility of using different and improved GAs to obtain better results. Improvements concerned the description of the pump conditions with a real number (and therefore in continuous form) and the introduction of elitism and of a slightly modified form of mutation. Simulations were obviously performed with reference to the same model under the same assumptions of the previous papers. Results showed significant improvements in the passage from a discrete to a continuous description of the pumps functioning and a slight improvement using elitism and no differences using mutation. The latter result might need some more research: mutation is introduced to enlarge the space in which the 'individuals' perform their search, and there is the need to understand whether this little improvement is due to the poor performance of this mutation or instead, because the space of search is already well defined. The need for more in-depth investigations is also investigated in the present paper.
\end{abstract}

Keywords: energy saving, genetic algorithms, optimization, water distribution.

\section{INTRODUCTION}

Water-distribution networks are a primary part of water supply systems, and, therefore, they represent one of the main infrastructure assets of the society, whose management must be effective, efficient and energy saving. To this end, hydraulic constraints are not sufficient to find the best system and to ensure that economic and energy conditions are taken into a full account. For this kind of complex and multi-objective optimization problem, the evolutionary computation (EC) procedure may be useful [1]. The procedure involves a large spectrum of heuristic approaches feasible to simulate the system evolution, which include: genetic algorithms (GAs) [2,3], evolutionary strategies [4,5], evolutionary programming [6] and genetic programming [7]. GAs are one of the most known ECs, because of their central use in water resources planning and management. They have been largely used in the last two decades to improve efficiency of water-distribution systems when traditional calculus-based and enumerative optimization methods showed that they were not able to cope with both the geometrical complexity of these systems (complex networks of pipes, junctions and hydraulic control elements) and the water demand of customers with required quality and affordable costs. One of the first water engineering applications of GAs was related to the optimization 
of pump schedules for a serial liquid pipeline [8,9]. GAs have been also used for least-cost design [10], and for network optimization [11-13].

Mackle et al. [14] were among the first to apply a binary GA to pump scheduling problems, to minimize energy costs, subject to reservoir filling and emptying constrains. Then Savic and Walters [10] developed a multi-objective GA (MOGA) approach to minimize both the energy costs and the number of pump switches. The first industrial application to the pump scheduling problem is reported by Atkinson et al. [15-17]. Van Zyl et al. [18] developed a hybrid optimization approach to reduce the excessive running times. Further improvements were provided by Prasad and Park [19], Farmani et al. [20] and Rao and Salomons [21].

The Authors [22,23] already developed a Simple GA for the optimization of pumps scheduling in a large town, and they applied it to the case of Milano, showing that large energy (and therefore economic) savings were possible. In the present work, developments of the initial simple GA have been implemented and the carried out results critically discussed to determine the possible path of further research for the network optimization.

\section{POSITION OF THE PROBLEM}

The problem is to guarantee a good level of service with the minimum energy expenditure. This is an optimization problem with constraints.

The objective function $(O F)$ to be minimized is the power required by the system $W$ (and consequently the required energy) which is obtained by summing the power required by each running pump; moreover, if the pressure at some control points falls below the value of $25 \mathrm{~m}$ (the minimum required by the service management), the value of the $O F$ is increased in function of the difference from the acceptable (MinPres) and the computed (CompPres) pressures to penalize the configuration; the $O F$ therefore becomes:

$$
\text { OF }=W \cdot 5 \cdot\left(1+\frac{\text { MinPres }- \text { CompPres }}{\text { MinPres }}\right) \quad \text { when CompPres }<\text { MinPres }
$$

the power $W$ is computed as:

$$
W=\frac{\gamma \cdot H \cdot Q}{\eta}
$$

where $\gamma$ is the specific weight of the fluid (water), $H$ is the head given by the pump for the discharge $Q$ and $\eta$ represents the efficiency of the pump. In all simulations, the efficiency has been accounted as constant in all situations and equal to 0.75 .

Moreover, in (1) MinPres and CompPres represent the minimum acceptable and the computed pressures, respectively, and MinPres $=25 \mathrm{~m}$; the $O F$ is minimized every full hour.

With regard to the constraints, the assumption is that pressures must be above (or equal) $25 \mathrm{~m}$, which are checked in some 'control points' located downstream the pumping stations. If the pressure in some control points falls outside the mentioned range, the value of $W$ is increased by a fixed value to penalize the carried out configuration; in the present form, if the pressure decreases below a fixed value of $1 \mathrm{~m}$ (i.e., pressure decreased to $24 \mathrm{~m}$ ), the $O F$ would be increased by $20 \%$. To solve this optimization problem GA procedures have been applied.

\section{SIMPLE GENETIC ALGORITHM}

The simple GA is based on the mechanics of natural selection and natural genetics, combining survival of the fittest among string structures with a structured yet randomized information 
exchange to form a search algorithm. In every generation, a new set of artificial 'individuals' (strings) is created using bits and pieces of the old. The method is sketched in Fig. 1.

The entire system is implemented with a binary string. Each pump is represented by 3 bits (i.e., possibilities) which describe its working status, i.e.: pump off, pump on working at 40 , $50,60,70,80,90$ and $100 \%$ of its nominal speed. On the all, the length of the string representing one scenario is composed by 96 (pumps) multiplied by 3 (bits) $=288$ values in $[0,1]$.

The first step in the implementation process of the GA consists in the generation of a number of configurations that will be used as the first generation of solutions ('population'): one of these is the actual configuration, the others are created randomly by the program.

Tests have been performed with different number of individuals which form the population. All the individuals that form the population are then simulated and their $W$ values computed, considered as the 'fitness' values of each individual.

These values are then used to favor the individuals that give better results in the 'reproduction' phase, that is, the creation of a new set of individuals that form the new population; the reproduction is carried out as a simple and quasi-random crossover between two different strings.

With regard to the initial population a variable number of individuals (within the range of 100-150) has been used; the number of iterations needed to reach the final configuration ranged between 100 and 200 .

Because of the partial randomness of the algorithm, each simulation may lead to a different result; therefore, for every hour of the day 10 simulations have been run, with different starting points.

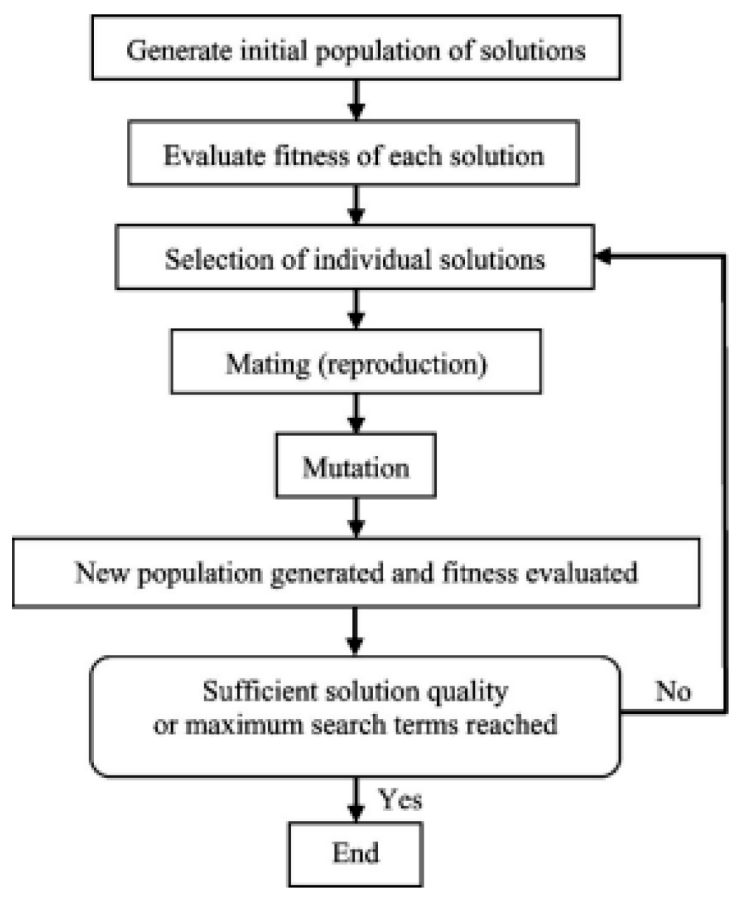

Figure 1: Generalized framework of the simple GA. 


\section{MODIFIED GENETIC ALGORITHM}

\subsection{Use of real values}

One of the main limitations of the procedure previously described consists in the discretization of the functioning of the pumps, which might be considered too broad. However, it is clear that the improvement of the discretization increases the dimensions of the problem with the further uncertainty of not knowing the appropriate level of discretization.

The modified GA utilizes evolutionary operators that are designed for both binary-coded chromosomes and real-valued variables without the need to use binary-coding for the latter $[24,25]$. In this paper, only the part related to the use of real values is applied: this means that the algorithm is applied exactly as previously described, but the string feasible to describe a single scenario is composed by 96 reals.

\subsection{Elitism}

At the beginning of each iteration, the fitness of the individuals are computed and sorted in descending order. The algorithm follows an elitist strategy, hence a constant fraction $f_{\text {elit }}$ of the population consisting of the fittest individuals are directly sent to the next generation. The rest of the population consisting of $1-f_{\text {elit }}$ are generated from reproduction (i.e., breeding) operations. In the literature is often let $f_{\text {elit }} \sim 1 \div 5 \%$, while in the paper is let $f_{\text {elit }}=6 \%$, when this procedure is applied. The aim of the procedure is obviously not to lose the best individuals.

\subsection{Mutation}

The procedure here developed is slightly different from the usual, as it previously selects a percentage of individuals for which one gene is randomly changed. The aim of this procedure is to avoid the GA to be trapped in local minima, letting the possibility to explore new zones in the searched area.

The implementation of this procedure consists of selecting the best individuals (following those selected by the elitism procedure, if applied); moreover it randomly chooses a position for the gene to be changed, which is substituted by a new one randomly generated. The constant fraction $f_{\text {muta }}$ to be mutated is let equal to $6 \%$, when this procedure is applied.

\section{CASE STUDY: THE MILANO WATER SUPPLY NETWORK}

The water supply system of Milano [22,23,26] acquires drinking water from a number of wells; pumps convey water to reservoirs located at the ground level. From those reservoirs, water is pumped directly in the network without the need for reservoirs located at higher altitude. The hydraulic head is therefore guaranteed by the pumping stations which action balances the effects of water demand, which pattern is shown in figure 2.

The pipelines have a total length of $2200 \mathrm{~km}$; in the network there are 31 pumping stations and in each of them 3-4 pumps are installed; each pump works with a discharge within the range $200-400 \mathrm{l} / \mathrm{s}$ and maximum head of $50 \mathrm{~m}$.

The model applied in the present research is that used for the previous investigations $[22,23]$ to guarantee consistency. Some simplifications have been introduced, which consist of taking into account only pipes with a diameter larger than $300 \mathrm{~mm}$, neglecting the smaller 


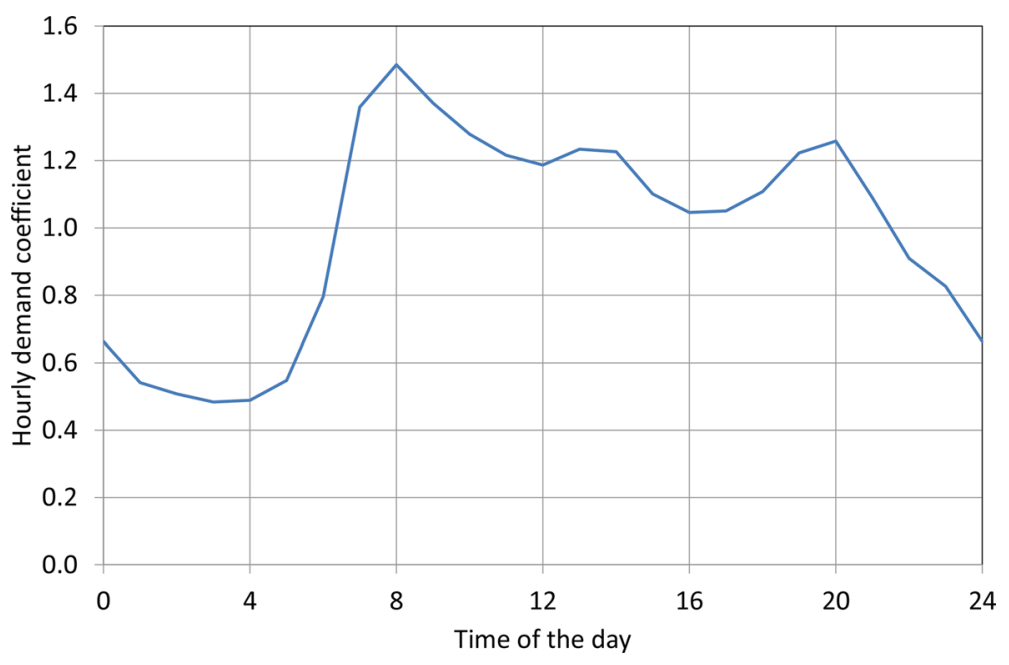

Figure 2: Pattern of the daily mean demand.

ones, with the aim of reducing the simulation times. However, fairly accurate reconstruction of the network was reached, which consisted of 4964 junction, 96 pumps, 26 stations with an overall length of pipes equal to $460 \mathrm{~km}$.

Results of the simulations have been compared with real data, recorded in the pumping stations and in some points of the network equipped with appropriate instrumentation.

Results confirmed [22,23] the possibility of saving energy and therefore economic savings both with and without the use of adjustable speed drivers, compared with the initial situation, which was determined out of the experience of the technicians.

In order to have an idea of the feasible economic gains, the energy cost has been let equal to $€ 0.10 / \mathrm{kWh}$ for the whole day. Therefore, considering the actual total energy in a year equal to $38.200 .000 \mathrm{kWh}$, with the use of ASD it is possible to save up to $7.000 .000 \mathrm{kWh} /$ year, which means an economic saving equal to $€ 700.000$.

\section{RESULTS}

Simulations have been performed using 100 individuals per population, and populations have been generated 100 times; each simulation has been performed 10 times because the method is partially random. As in the previous work, the optimization is run each full hour.

In Fig. 3, the values carried out for a simulated case are reported (in Fig.:1 p.m., best result obtained with real inverter, elitism and no mutation). As can be seen, there is a large almost immediate improvement; then, improvements become smaller but they continue until all the individuals converge to the same (best) value. Spikes, visible in the figure, are due to solutions which are considered unacceptable and therefore penalized.

Results show a large improvement in the results, when the real values are used to describe the pumps working conditions, using ASD, with respect to their discrete Boolean description. As can be seen in Table 1 (and Fig. 4), with respect to the results carried out in our previous paper, a further improvement of about $20 \%$ is still possible. Figure 4 shows that these improvements are possible during the hours of the day when more energy is required.

Elitism allows another small improvement, in terms of few percent (see Table 1, which shows a further improvement of about $5 \%$ ). This is probably due to a faster achievement of 


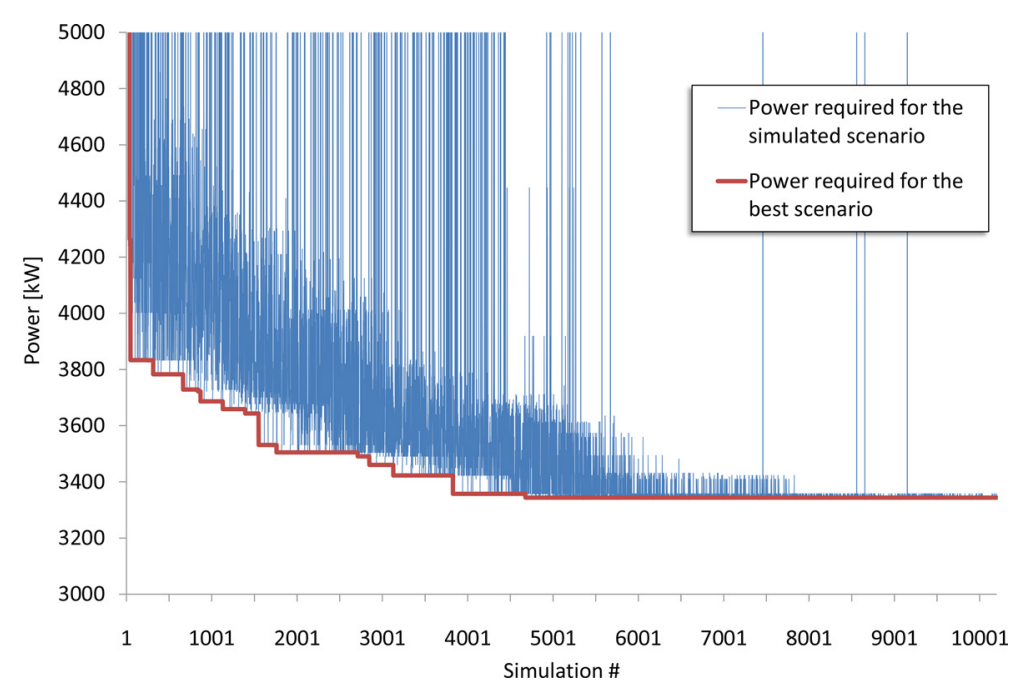

Figure 3: Values of the power required in the different scenarios during optimization.

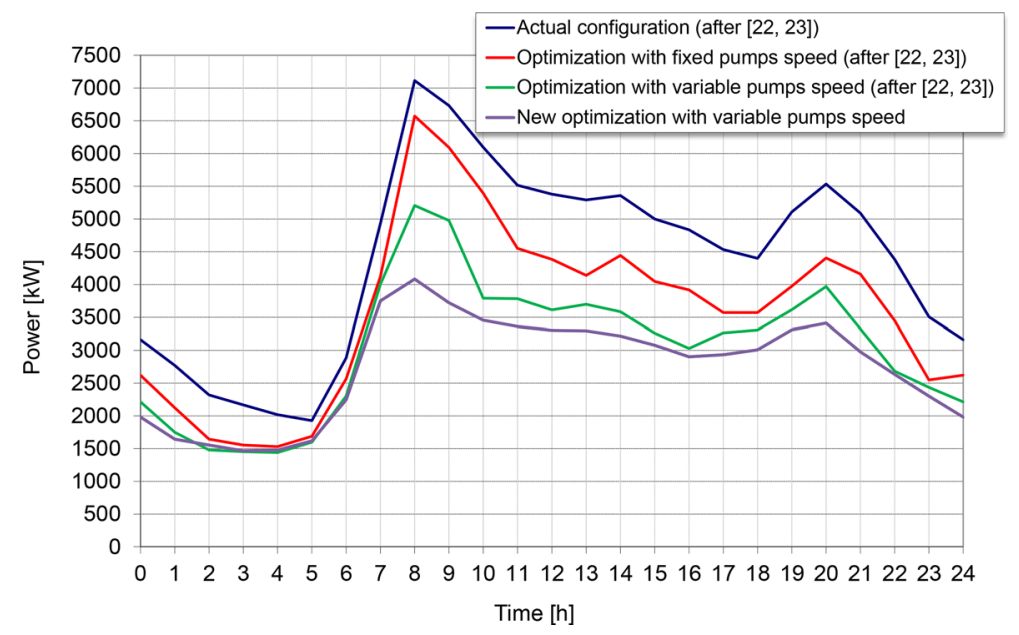

Figure 4: Required power in an average day: improvements with the new methods.

the best result: in other terms, there is the possibility to reach the same value even without the elitism procedure, but using a higher number of iteration.

On the other hand, mutation allows no improvements: as can be seen in Table 1, the few points of percentage in difference respect the values already reached, show improvement or worsening, and this is tied to the randomness of the procedure. This probably means that the search space is already well evaluated - or the implemented procedure is unable to escape local minima.

However, with the optimization carried out by the use of real values and adding elitism, the yearly energy required is equal to $22.800 .000 \mathrm{kWh}$, that means a global economic saving equal to $€ 1.5$ million per year. 
Table 1: Energy required, using different optimization methods, for each hour of the average day.

\begin{tabular}{|c|c|c|c|c|c|c|c|c|c|c|}
\hline \multirow[b]{2}{*}{ Time } & \multicolumn{4}{|c|}{ After $[22,23]$} & \multicolumn{6}{|c|}{ Improved GA } \\
\hline & $\begin{array}{l}\text { Actual } \\
{[\mathrm{kW}]}\end{array}$ & $\begin{array}{l}\text { Fix } \\
{[\mathrm{kW}]}\end{array}$ & $\begin{array}{l}\text { ASD } \\
{[\mathrm{kW}]}\end{array}$ & $\begin{array}{l}\text { ASD Vs } \\
\text { Actual } \\
{[\%]}\end{array}$ & $\begin{array}{l}\text { Real } \\
{[\mathrm{kW}]}\end{array}$ & $\begin{array}{l}\text { Real Vs } \\
A S D[\%]\end{array}$ & $\begin{array}{l}\text { Elitism } \\
{[\mathrm{kW}]}\end{array}$ & $\begin{array}{l}\text { Elitism } \\
\text { Real[\%] }\end{array}$ & $\begin{array}{l}\text { sMutat. } \\
{[\mathrm{kW}]}\end{array}$ & $\begin{array}{l}\text { Mutat. Vs } \\
\text { Elitism } \\
{[\%]}\end{array}$ \\
\hline 0 & 3161 & 2650 & 2459 & 22.20 & 1980 & 19.49 & 1831 & 7.52 & 1777 & 2.97 \\
\hline 1 & 2769 & 2124 & 1980 & 28.49 & 1642 & 17.07 & 1502 & 8.54 & 1504 & -0.16 \\
\hline 2 & 2320 & 1643 & 1619 & 30.22 & 1554 & 4.00 & 1441 & 7.30 & 1447 & -0.45 \\
\hline 3 & 2167 & 1677 & 1468 & 32.24 & 1465 & 0.20 & 1377 & 6.01 & 1359 & 1.33 \\
\hline 4 & 2018 & 1593 & 1463 & 27.50 & 1475 & -0.84 & 1358 & 7.92 & 1342 & 1.20 \\
\hline 5 & 1927 & 1805 & 1699 & 11.82 & 1618 & 4.76 & 1564 & 3.37 & 1532 & 2.03 \\
\hline 6 & 2884 & 2764 & 2764 & 4.16 & 2243 & 18.85 & 2200 & 1.90 & 2123 & 3.53 \\
\hline 7 & 4930 & 4901 & 4615 & 6.39 & 3750 & 18.75 & 3485 & 7.07 & 3527 & -1.20 \\
\hline 8 & 7113 & 6623 & 5743 & 19.26 & 4083 & 28.90 & 3930 & 3.75 & 4007 & -1.96 \\
\hline 9 & 6733 & 6096 & 5153 & 23.48 & 3721 & 27.78 & 3611 & 2.98 & 3563 & 1.32 \\
\hline 10 & 6095 & 5394 & 5009 & 17.81 & 3455 & 31.02 & 3370 & 2.47 & 3231 & 4.14 \\
\hline 11 & 5515 & 4552 & 4550 & 17.51 & 3359 & 26.17 & 3184 & 5.21 & 3121 & 1.99 \\
\hline 12 & 5378 & 4535 & 4535 & 15.67 & 3304 & 27.15 & 3134 & 5.14 & 2998 & 4.34 \\
\hline 13 & 5291 & 4715 & 4548 & 14.04 & 3297 & 27.52 & 3159 & 4.18 & 3180 & -0.66 \\
\hline 14 & 5360 & 4528 & 4513 & 15.80 & 3217 & 28.71 & 3160 & 1.77 & 3105 & 1.73 \\
\hline 15 & 5001 & 4175 & 4213 & 15.76 & 3076 & 26.99 & 2776 & 9.74 & 2839 & -2.27 \\
\hline 16 & 4835 & 3918 & 3918 & 18.97 & 2902 & 25.93 & 2754 & 5.10 & 2770 & -0.57 \\
\hline 17 & 4534 & 3781 & 3725 & 17.85 & 2933 & 21.26 & 2670 & 8.97 & 2677 & -0.27 \\
\hline 18 & 4400 & 3710 & 3627 & 17.56 & 3006 & 17.12 & 2928 & 2.61 & 2901 & 0.92 \\
\hline 19 & 5108 & 4371 & 4133 & 19.10 & 3311 & 19.89 & 3076 & 7.09 & 3179 & -3.35 \\
\hline 20 & 5534 & 4875 & 4716 & 14.79 & 3411 & 27.66 & 3269 & 4.17 & 3149 & 3.67 \\
\hline 21 & 5091 & 4162 & 4229 & 16.93 & 2977 & 29.60 & 2949 & 0.97 & 2909 & 1.33 \\
\hline 22 & 4385 & 3450 & 3267 & 25.49 & 2634 & 19.37 & 2398 & 8.98 & 2368 & 1.22 \\
\hline 23 & 3508 & 2838 & 2696 & 23.17 & 2301 & 14.65 & 2205 & 4.16 & 2199 & 0.26 \\
\hline & & & & 19.01 & & 20.08 & & 5.29 & & 0.88 \\
\hline
\end{tabular}

\section{CONCLUSIONS}

In previous papers, a simple GA was developed for the optimization of pumps functioning in water-distribution networks; its application at the water supply network of Milano showed the possibility for a great improvement of network's performance in terms of energy and economic savings. In the present paper, the possibility of using different and improved GAs to obtain better results has been investigated. These improvements concern the description of the pump conditions with real number (and therefore in continuous form) and the possibility of introducing elitism and a slightly modified form of mutation. Simulations were carried out with regard to the same model applied in previous investigations, under the same assumptions. Results show a significant improvement in the passage from discrete to continuous 
description of the pumps functioning, a slight improvement using elitism and no differences using mutation.

The latter result might need more research: mutation is introduced to enlarge the space in which the 'individuals' perform their search, and there is the need to understand whether this little improvement is due to the poor performance of this mutation or, instead, because the space of search is already well defined.

It should also be verified whether the GAs bring to the real maxima; it is believed that they are able to reach points very close to the maximum, but not the actual maximum itself. This could be verified by using an hill climbing procedure to the carried out scenarios.

Moreover, this latter procedure could clarify whether the GAs lead to a single maximum, or many local maxima exist bringing different scenarios with very similar energy consumption. In the latter case, it would be possible to combine the savings of the energy with the different requests of a complex network as that of Milano (i.e., pumping better water quality water, preferring some wells and discarding others; obtain the solution which allows strategic reservoirs to be filled when demands are higher; ...), with targets that might be contradictory and, therefore, that require to move on the Pareto boundary.

\section{ACKNOWLEDGMENT}

The authors wish to acknowledge Ms Grazia Mele for carrying out all the many simulations required to make this work.

\section{REFERENCES}

[1] Back, T., Fogel, D.B. \& Michalewicz, Z. (eds.), Handbook of Evolutionary Computation, Institute of Physics publishing \& Oxford University Press: New York, 1997.

[2] Holland, J.H., Outline for a logical theory of adaptive systems. Journal of The Association for Computing Machinery, 3, pp. 297-314, 1962.

http://dx.doi.org/10.1145/321127.321128

[3] Holland, J.H., Adaptation in Natural and Artificial Systems, University of Michigan Press: Ann Arbor, Mich., 1975.

[4] Rechenberg, I., Evolutionstrategie: optimierung technisher systeme nach prinzipien der biologischen evolution. Frommann-Hoolzboog Verlag: Stuttgart, 1973. (in German)

[5] Schewefel, H.P., Numerical Optimization of Computer Models, John Wiley and Sons: New York, 1981.

[6] Fogel, L., Owens, A. \& Walsh, M., Artificial Intelligence Through Simulated Evolution, John Wiley \& Sons, Inc.: New York, 1966Koza, 1992.

[7] Goldberg, D.E., Genetic Algorithms in Search, Optimization and Machine Learning. Addison-Wesley: Reading, MA, 1989.

[8] Golberg, D.E. \& Kuo, C.H. Genetic algorithms in pipeline optimization. Iournal of Computing in Civil Engineering, 1(2), pp. 128-141, 1987. http://dx.doi.org/10.1061/(ASCE)0887-3801(1987)1:2(128)

[9] Simpson, A.R., Dandy, G.C. \& Murphy, L.J., Genetic algorithms compared to other techniques for pipe optimization. Journal of Water Resources Planning and Management, ASCE, 120(4), pp. 423-443, 1994. http://dx.doi.org/10.1061/(ASCE)0733-9496(1994)120:4(423)

[10] Savic, D.A. \& Walters, G.A., Genetic algorithm for least-cost design of water distribution networks. Journal of Water Resources Planning and Management, ASCE, 123, pp. 67-77, 1997.

http://dx.doi.org/10.1061/(ASCE)0733-9496(1997)123:2(67) 
[11] Alperovits, E. \& Shamir, U., Design of optimal water distribution systems. Water Resources Research, 13(6), pp. 885-900, 1977. http://dx.doi.org/10.1029/WR013i006p00885

[12] Fujiwara, O. \& Khang, D.B., A two-phase decomposition method for optimal design of looped water distribution networks. Water Resources Research, 26(4), pp. 539-549, 1990. http://dx.doi.org/10.1029/WR026i004p00539

[13] Schaake, J.C. \& Lai, D., Linear Programming and Dynamic Programming Application to Water Distribution Network Design. Rep. No. 116, Department of Civil Engineering, MIT, Cambridge, 1969.

[14] Mackle, G., Savic, D.A. \& Walters, G.A. Application of genetic algorithms to pump scheduling for water supply. Proceedings of Genetic Algorithms in Engineering Systems: Innovations and Applications, GALESIA '95, IEE, London, pp. 400-405, 1995.

[15] Atkinson, R., van Zyl, J.E., Walters, G.A. \& Savic, D.A., Genetic algorithm optimization of level-controlled pumping station operation. Water Network Modelling for Optimal Design and Management, Centre for Water Systems, Exeter, UK, pp. 79-90, 2000.

[16] De Schaetzen, W.B.F., Savic, D.A. \& Waltres, G.A. A genetic algorithm approach to pump scheduling in water supply system. Hydroinformatics, 1998.

[17] Illich, N. \& Simovic, S.P., Evolutionary algorithm for minimization of pumping cost. Journal of Computing in Civil Engineering, ASCE, 12, pp. 232-240, 1998. http://dx.doi.org/10.1061/(ASCE)0887-3801(1998)12:4(232)

[18] van Zyl, J., Savic, D.A. \& Walters, G.A., Operational optimization of water distribution systems using a hybrid genetic algorithm method. Journal of Water Resources Planning and Management, 130(2), pp. 160-170, 2004. http://dx.doi.org/10.1061/(ASCE)0733-9496(2004)130:2(160)

[19] Prasad, T. D. \& Park, N.S., Multiobjective genetic algorithms for design of water distribution networks. Journal of Water Resources Planning and Management, 130(1), pp. 73-82, 2004. http://dx.doi.org/10.1061/(ASCE)0733-9496(2004)130:1(73)

[20] Farmani, R., Savic, D.A. \& Walters, G.A., Evolutionary multi-objective optimization in water distribution network design. Engineering Optimization, 37(2), pp. 167-183, 2005. http://dx.doi.org/10.1080/03052150512331303436

[21] Rao, Z. \& Salomons, E., Development of a real-time, near optimal control process for water-distribution networks. Journal of Hydroinformatics, 9(1), pp. 25-37, 2007. http://dx.doi.org/10.2166/hydro.2006.015

[22] Mambretti, S., Optimization of the pumping station of Milano water supply network with genetic algorithms, 3rd International Conference on Energy and Sustainability, Alicante, Spain, pp. 185-194, 11-13 April 2011.

[23] Mambretti, S. \& Orsi, E., Optimization of Pumping Stations in Complex Water Supply Networks through Evolutionary Computation Methods, Accepted for publication in the Journal of American Water Works Association, scheduled for February 2016. http://dx.doi.org/10.2495/esus110161

[24] Mundo, D. \& Yan, H.S., Kinematic optimization of ball-screw transmission mechanisms. Mechanism and Machine Theory, 42, pp. 34-47, 2007. http://dx.doi.org/10.1016/j.mechmachtheory.2006.02.002 
[25] Behandish, M. \& Wu, Z.Y., Concurrent pump scheduling and storage level optimization using meta-models and evolutionary algorithms. Procedia Engineering, 70, pp. 103112, 2014.

http://dx.doi.org/10.1016/j.proeng.2014.02.013

[26] Motta, V., L'acquedotto di Milano, Comune di Milano, 1989. (in Italian) 